\title{
Efficiency of ultrafast optically induced spin transfer in Heusler compounds
}

\author{
Daniel Steil $\odot,{ }^{1, *}$ Jakob Walowski $\odot,{ }^{2}$ Felicitas Gerhard, ${ }^{3}$ Tobias Kiessling, ${ }^{3}$ Daniel Ebke, ${ }^{4}$ Andy Thomas, ${ }^{5}$ \\ Takahide Kubota $\odot,{ }^{6,7}$ Mikihiko Oogane, ${ }^{8,7}$ Yasuo Ando, ${ }^{8,7}$ Johannes Otto $\odot,{ }^{1}$ Andreas Mann, ${ }^{1}$ Moritz Hofherr $\odot,{ }^{9}$ \\ Peter Elliott $\odot,{ }^{10}$ John Kay Dewhurst, ${ }^{11}$ Günter Reiss, ${ }^{4}$ Laurens Molenkamp $\odot{ }^{3}$, Martin Aeschlimann $\odot,{ }^{9}$ Mirko Cinchetti $\odot,{ }^{12}$ \\ Markus Münzenberg $\odot{ }^{2}$ Sangeeta Sharma, ${ }^{10}$ and Stefan Mathias $\odot{ }^{1,13, \dagger}$ \\ ${ }^{1}$ I. Physikalisches Institut, Universität Göttingen, 37077 Göttingen, Germany \\ ${ }^{2}$ Institut für Physik, Universität Greifswald, 17489 Greifswald, Germany \\ ${ }^{3}$ Physikalisches Institut (EP3) der Universität Würzburg, 97074 Würzburg, Germany \\ ${ }^{4}$ Department of Physics, Bielefeld University, 33615 Bielefeld, Germany \\ ${ }^{5}$ Leibniz Institute for Solid State and Materials Research Dresden (IFW Dresden), Institute for Metallic Materials, \\ Helmholtzstrasse 20, 01069 Dresden, Germany \\ ${ }^{6}$ Institute for Materials Research, Tohoku University, Sendai 980-8577, Japan \\ ${ }^{7}$ Center for Spintronics Research Network, Tohoku University, Sendai 980-8577, Japan \\ ${ }^{8}$ Department of Applied Physics, Tohoku University, Sendai 980-8579, Japan \\ ${ }^{9}$ Department of Physics and Research Center OPTIMAS, University of Kaiserslautern, \\ Erwin-Schrödinger-Strasse 46, 67663 Kaiserslautern, Germany \\ ${ }^{10}$ Max-Born-Institut für Nichtlineare Optik und Kurzzeitspektroskopie, Max-Born-Strasse 2A, 12489 Berlin, Germany \\ ${ }^{11}$ Max-Planck-Institut für Mikrostrukturphysik, Weinberg 2, 06120 Halle, Germany \\ ${ }^{12}$ Experimentelle Physik VI, TU Dortmund, 44221 Dortmund, Germany \\ ${ }^{13}$ International Center for Advanced Studies of Energy Conversion (ICASEC), Universität Göttingen, 37077 Göttingen, Germany
}

(Received 24 October 2019; accepted 16 April 2020; published 20 May 2020)

\begin{abstract}
Optically induced spin transfer (OISTR) is a pathway to control magnetization dynamics in complex materials on femto- to attosecond timescales. The direct interaction of the laser field with the material creates transient nonequilibrium states, which can exhibit an efficient spin transfer between different magnetic subsystems. How far this spin manipulation via OISTR is a general phenomenon or restricted to a subset of materials with specific properties is an open experimental and theoretical question. Using time-resolved magneto-optical Kerr measurements and time-dependent density functional theory we investigate OISTR in Heusler compounds. We show that the half-Heusler materials NiMnSb and CoMnSb exhibit strong signatures of OISTR, whereas this is less pronounced in the full-Heusler compounds $\mathrm{Co}_{2} \mathrm{MnSi}, \mathrm{Co}_{2} \mathrm{FeSi}$, and $\mathrm{Co}_{2} \mathrm{Fe} \mathrm{Al}$ in agreement with $a b$ initio calculations. Our work opens up a systematic path for coherent manipulation of spin dynamics by direct light-matter interaction.
\end{abstract}

DOI: 10.1103/PhysRevResearch.2.023199

\section{INTRODUCTION}

The manipulation of the magnetic state in solids by light has created a new paradigm in condensed matter physics, where research has been driven by the prospect of future applications as well as the fundamental question of how fast the magnetic state in a solid can be controlled. Here, recent publications have verified the theoretically predicted [1-3] ultrafast optically induced spin transfer (OISTR) effect [4-6], where the manipulation of spin dynamics is only limited by the pulse length of the shortest light pulses that have been

\footnotetext{
*dsteil@gwdg.de

†smathias@uni-goettingen.de

Published by the American Physical Society under the terms of the Creative Commons Attribution 4.0 International license. Further distribution of this work must maintain attribution to the author(s) and the published article's title, journal citation, and DOI.
}

created so far. If a solid is strongly excited with a light pulse, optical transitions create a transient nonequilibrium electron distribution. In complex compounds, alloys, and multilayer structures, where the band structure consists of orbital contributions from different elements, certain optical transitions support in principle a transfer of spin momentum from one subsystem to another, which is the essence of OISTR.

In order to elucidate whether this recently found OISTR effect is a general phenomenon that is efficient enough to be used to manipulate spin dynamics on the fastest timescales, we investigate a set of Heusler materials. Heuslers are ideally suited for the study of OISTR, because most compounds are multisublattice ferromagnets with several independently active magnetic sites in their atomic structure. Moreover, via control of the elemental composition of Heusler compounds, a wide range of physical properties such as half metallicity, topological effects, multiferroicity, and their use in spintronics can be realized [7-9].

In this paper, we show that this tunability enables us to manipulate ultrafast magnetization dynamics in Heusler 
compounds via material composition, where the efficiency of OISTR between different elemental magnetic moments can be amplified or suppressed. We study the ultrafast magnetization dynamics of the half-Heusler compounds $\mathrm{NiMnSb}$ and $\mathrm{CoMnSb}$ and of the full Heuslers $\mathrm{Co}_{2} \mathrm{MnSi}, \mathrm{Co}_{2} \mathrm{FeSi}$, and $\mathrm{Co}_{2} \mathrm{FeAl}$. Experimentally, we find that the half-Heusler systems show a strong signature of OISTR, i.e., an initial increase of the MOKE signal, whereas the full Heuslers show a less pronounced OISTR effect. Our findings are corroborated by theoretical calculations, and we connect amplification and suppression of OISTR to specific properties of the band structure of the investigated Heuslers. We thus present a systematic pathway for an efficient control of spin dynamics on the fastest timescales that are reachable today.

\section{EXPERIMENTAL DETAILS}

The experimental data shown in this paper have been collected by two teams with two similar setups that make use of the time-resolved magneto-optical Kerr effect (TR-MOKE). $\mathrm{CoMnSb}$ and $\mathrm{Co}_{2} \mathrm{FeAl}$ were measured in a monochromatic TR-MOKE setup with a central wavelength of $800 \mathrm{~nm}$ for both the pump and the probe beam ("MOKE setup 1"). The laser source is a regenerative amplifier system (Coherent RegA) with a repetition rate of $250 \mathrm{kHz}$, a pulse energy of about $1 \mu \mathrm{J} /$ pulse, and 50 fs pulse duration (for details cf. Refs. [10-12]). The $\mathrm{NiMnSb}$ and $\mathrm{Co}_{2} \mathrm{FeSi}$ data were recorded in a bichromatic TR-MOKE scheme with a pump wavelength of $800 \mathrm{~nm}$ and $400 \mathrm{~nm}$ probe wavelength. The laser source here is a multipass amplifier (Quantronix Odin; "MOKE setup 2") with a repetition rate of $1 \mathrm{kHz}$, a pulse energy of about $1 \mathrm{~mJ} /$ pulse, and $60 \mathrm{fs}$ pulse duration (for details cf. Refs. [13-15]). For comparison, $\mathrm{Co}_{2} \mathrm{MnSi}$ samples were studied with both setups. Further information on the sample systems is given in the Supplemental Material [16].

\section{THEORETICAL BACKGROUND}

Theoretical simulations were performed in two steps: First the ground state of all the materials was calculated using density functional theory (DFT). This ground state was then simulated to be pumped with a laser and the dynamics of the density and the magnetization density were studied using a time-dependent extension of DFT (TD-DFT). TD-DFT maps the difficult problem of calculating a system of interacting electrons to a more computationally tractable system of noninteracting electrons. This fictitious system, the Kohn-Sham (KS) system, is defined such that it reproduces the density evolution of the interacting system. Knowledge of this density is sufficient in order to extract all observables of the system [17]. To study spin dynamics, the KS equation to be propagated is

$$
\begin{aligned}
i \frac{\partial \phi_{j}(\mathbf{r}, t)}{\partial t}= & \left\{\frac{1}{2}\left[-i \nabla+\frac{1}{c} \mathbf{A}_{\mathrm{ext}}(t)\right]^{2}\right. \\
& +v_{s}(\mathbf{r}, t)+\frac{1}{2 c} \boldsymbol{\sigma} \cdot \mathbf{B}_{s}(\mathbf{r}, t) \\
& \left.+\frac{1}{4 c^{2}} \boldsymbol{\sigma} \cdot\left[\nabla v_{s}(\mathbf{r}, t) \times-i \nabla\right]\right\} \phi_{j}(\mathbf{r}, t),
\end{aligned}
$$

where $\phi_{j}(\mathbf{r}, t)$ are two-component Pauli spinors, $\mathbf{A}_{\mathrm{ext}}(t)$ is the external laser field, written as a purely time-dependent vector potential, $\sigma$ are the Pauli matrices, $v_{s}(\mathbf{r}, t)=v_{\text {ext }}(\mathbf{r})+$ $v_{\mathrm{H}}(\mathbf{r}, t)+v_{\mathrm{xc}}(\mathbf{r}, t)$ is the $\mathrm{KS}$ effective scalar potential, and $\mathbf{B}_{s}(\mathbf{r}, t)=\mathbf{B}_{\text {ext }}(\mathbf{r}, t)+\mathbf{B}_{\mathrm{xc}}(\mathbf{r}, t)$ is the $\mathrm{KS}$ effective magnetic field. These effective potentials ensure that the dynamics of the density and magnetization density match that of the interacting system. The problem is defined by the external potentials, where the external scalar potential $v_{\text {ext }}(\mathbf{r})$ includes the electron-nuclei interaction, while $\mathbf{B}_{\text {ext }}(\mathbf{r}, t)$ is any external magnetic field which interacts with the electronic spins via the Zeeman interaction. The Hartree potential, $v_{\mathrm{H}}(\mathbf{r}, t)$, is the classical electrostatic interaction. Finally, we have the exchange-correlation (XC) potentials, the scalar $v_{\mathrm{xc}}(\mathbf{r}, t)$ and the $\mathrm{XC}$ magnetic field $\mathbf{B}_{\mathrm{xc}}(\mathbf{r}, t)$, which require approximation [18-20]. The last term in Eq. (1) is the spin-orbit coupling term. In what follows a fully noncollinear spin-dependent version of this equation is employed [21,22]. All calculations are performed using the ELK electronic structure code [23]. The Brillouin zone was sampled with a $8 \times 8 \times 8$ mesh. The $\mathrm{XC}$ energy functional was LDA, which was extended to treat noncollinear systems using the method of Kübler [24]. For time propagation, a time step of 2.42 as was used [22].

\section{RESULTS AND DISCUSSION}

Figure 1 shows the magnetization dynamics of the half Heuslers CoMnSb and NiMnSb [Fig. 1(a)] versus the magnetization dynamics of the full Heuslers [Fig. 1(b)]. Clearly, the two half-Heusler materials show a pronounced initial increase of the magnetization, which is a strong indication for an OISTR process [4,5]. At later times ( $t>200 \mathrm{fs})$, secondary scattering and transport processes lead to the usual demagnetization [25-28]. In contrast, the full Heuslers [Fig. 1(b)] show no evidence for OISTR in our experiment, only the usual demagnetization as found in the literature [11,13-15,29-32]. Apparently, the efficiency of OISTR depends on the elemental composition and the magnetic moments of the different sublattices in the Heusler compounds.

We now turn to TD-DFT to elucidate how the material's composition controls the efficiency of OISTR in the half-Heusler versus the full-Heusler compounds. Figure 2(a) shows the calculated total (black line) and $d$-projected density of states (DOS) of Co (blue) and Mn (pink) for the half Heusler CoMnSb. In order to support an OISTR process, it is necessary to efficiently pump an optical transition that predominantly transfers majority and/or minority spins from one sublattice to another. This can easily be achieved, if the DOS above and below $E_{\mathrm{F}}$ of the two subsystems significantly differs.

In a toy model, the ideal "OISTR material" would, for the first elemental subsystem, exhibit a large DOS below $E_{\mathrm{F}}$ solely in the minority channel, and a large DOS above $E_{\mathrm{F}}$ solely in the majority channel. For the second element, the situation should be vice versa, so that every spin-conserving optical transition pumps spins from one elemental subsystem to the other. As the magnetization is the imbalance between minority and majority spins, such spin-transfer processes directly manipulate the magnetization in the subsystems, while the total magnetization remains constant. The probability for 


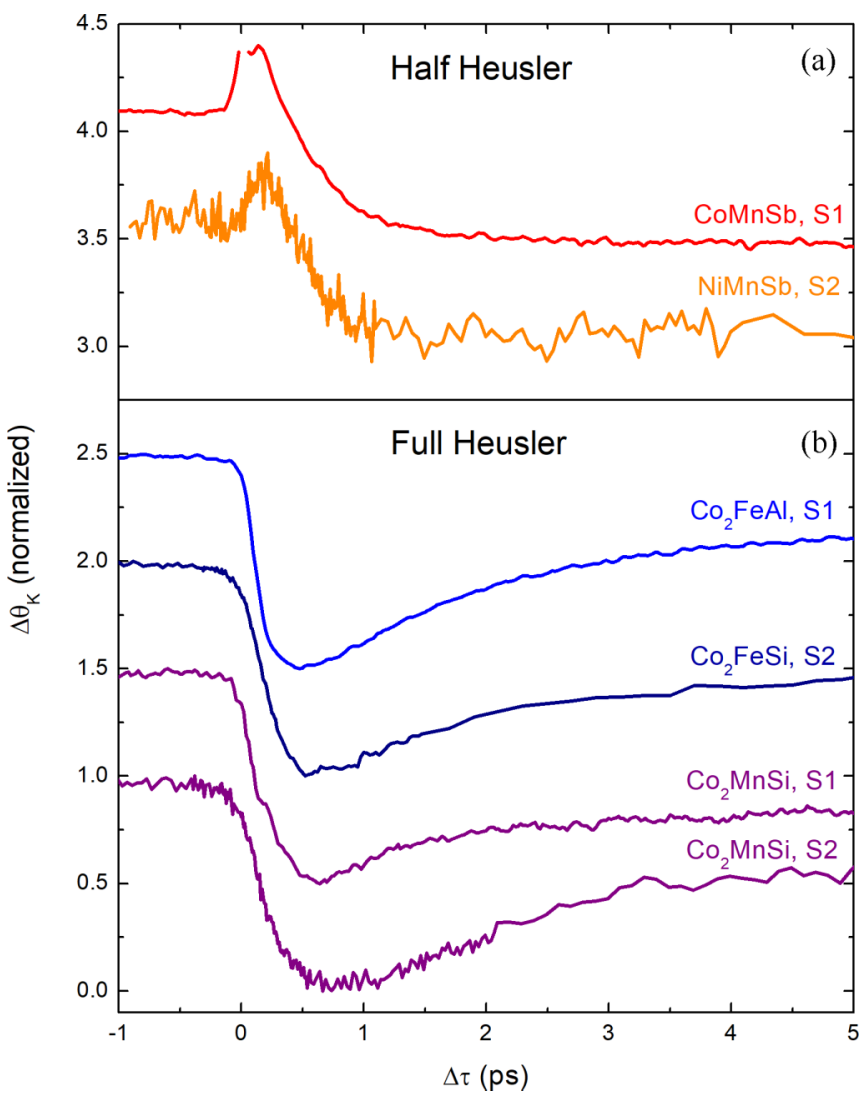

FIG. 1. (a) Experimentally observed magnetization dynamics in the half-Heusler compounds CoMnSb and $\mathrm{NiMnSb}$ and the (b) fullHeusler compounds $\mathrm{Co}_{2} \mathrm{FeAl}, \mathrm{Co}_{2} \mathrm{FeSi}$, and $\mathrm{Co}_{2} \mathrm{MnSi}$ investigated using the transient change in Kerr signal. Both half Heuslers show a pronounced increase in the magnetization upon excitation within the first $\sim 100 \mathrm{fs}$. This feature is not observed in the full Heuslers. Data are normalized between 0 and 1 and shifted for better visibility. S1 and S2 denote measurements with MOKE setup 1 and MOKE setup 2, respectively. Data for $\mathrm{Co}_{2} \mathrm{MnSi} \mathrm{S} 2$ from Ref. [15].

the spin-transfer processes additionally depends on the matrix elements for the respective optical transitions. However, we will see that arguments based on the DOS in the minority and majority channels already provide a good estimate of the appearance and efficiency of OISTR in Heusler compounds.

We start with the half Heusler CoMnSb, which shows a pronounced signature of OISTR in the TR-MOKE data [Fig. 1(a)]. If we compare the projected DOS of Co (blue) and Mn (pink) in Fig. 2(a) in the energetic range of possible optical transitions with our pump pulse $\left(1.5 \mathrm{eV}\right.$ above and below $\left.E_{\mathrm{F}}\right)$ we find that the DOS is quite similar for both elements in the majority channel. However, the minority channel shows significant differences: Below $E_{\mathrm{F}}$, the projected DOS of Co is larger than the projected DOS of Mn. Above $E_{\mathrm{F}}$, we find the opposite situation; i.e., the projected DOS of Co is extremely low, while the projected DOS of Mn is large, and we mark the difference in Fig. 2(a) in yellow for better visibility. Optical transitions in the minority channel therefore predominantly occur between the peaks in the occupied projected DOS of Co and the peaks in the unoccupied projected DOS of Mn, which are connected by the photon energy of $1.5 \mathrm{eV}$ (red arrow). Hence, an efficient spin transfer arises between the two magnetic sublattices. In terms of magnetization, counting the number of majority versus minority electrons, we pump minority electrons from $\mathrm{Co}$ to $\mathrm{Mn}$, which increases the magnetization in Co and decreases the magnetization in $\mathrm{Mn}$.

In order to corroborate whether the proposed OISTR process is active in CoMnSb, we turn our focus to the TDDFT calculations shown in Fig. 2(b) depicting the calculated relative change of the magnetization for the subsystems of Co and Mn. Clearly, the magnetization in Co increases up to a value of $\sim 550 \%$, which corresponds to an increase of its magnetic moment of about $+0.25 \mu_{\mathrm{B}}$ /atom. Simultaneously, the $\mathrm{Mn}$ magnetization decreases; however, the relative change is much less pronounced, since the total moment on the Mn sublattice of CoMnSb is about $3.2 \mu_{\mathrm{B}}$ /atom and therewith 68 times higher than the total moment in the Co sublattice with only $0.047 \mu_{\mathrm{B}} /$ atom.

The situation for the second half Heusler NiMnSb, which also shows strong OISTR in the experiment [Fig. 1(a)], is alike, with an absolute moment transfer of $+0.18 \mu_{\mathrm{B}} /$ atom from $\mathrm{Mn}$ to $\mathrm{Ni}$, corresponding to a relative increase of the $\mathrm{Ni}$ magnetization of about $65 \%$ (the initial $\mathrm{Ni}$ moment is $0.28 \mu_{\mathrm{B}} /$ atom). The theoretical data are shown in the Supplemental Material [16].

Note that the peaklike structure at 40 fs for Co in Fig. 2(b) cannot be resolved experimentally due to limited time resolution. The experiment, nevertheless, clearly captures the averaged OISTR-induced increase within the first 100 fs. Thereafter, the experimental signal is diminished due to the usual demagnetization, e.g., by secondary processes such as thermalization of hot electrons with the lattice (ElliotYafet scattering) [25]. This demagnetization on much longer timescales [Fig. 1(a)], on the other hand, is not captured by TD-DFT, where secondary demagnetization processes are not taken into account and the signal after about $100 \mathrm{fs}$ persists for any longer time frame simulated. Also, because the experimental data are measured at room temperature and the theoretical data are calculated at $T=0 \mathrm{~K}$, a full quantitative comparison is not currently feasible. Nevertheless, the important observation here, i.e., the ultrafast increase caused by OISTR, is clearly visible both in the experimental data as well as in theory.

Next, we want to focus on the differences between the half-Heusler and the full-Heusler materials. Figure 2(c) shows the calculated total (black) and $d$-projected DOS of Fe (red) and $\mathrm{Co}$ (blue) in $\mathrm{Co}_{2} \mathrm{FeSi}$. Evidently, there is still a difference between the projected DOS in the minority channel, marked once again in yellow for the most relevant $1.5 \mathrm{eV}$ transitions from a large DOS in the occupied to a large DOS in the unoccupied regime. However, this difference is less pronounced in comparison to the half Heusler; see Fig. 2(a). In particular, we find an increased number of Co states in the minority channel above $E_{\mathrm{F}}$. In consequence, we expect OISTR to be less efficient in the full Heusler. Indeed, this behavior is confirmed by the TD-DFT; see Fig. 2(d), blue line. Here, the maximum relative increase of magnetization for Co is only $\sim 5 \%$, which corresponds to an absolute moment transfer of $0.05 \mu_{\mathrm{B}} /$ atom for an initial moment of about $1.15 \mu_{\mathrm{B}} / \mathrm{Co}$-atom. Having a look at the other full Heuslers $\mathrm{Co}_{2} \mathrm{FeAl}$ and $\mathrm{Co}_{2} \mathrm{MnSi}$, the situation is once again similar; see Fig. 3. 


\section{Half Heusler: CoMnSb}

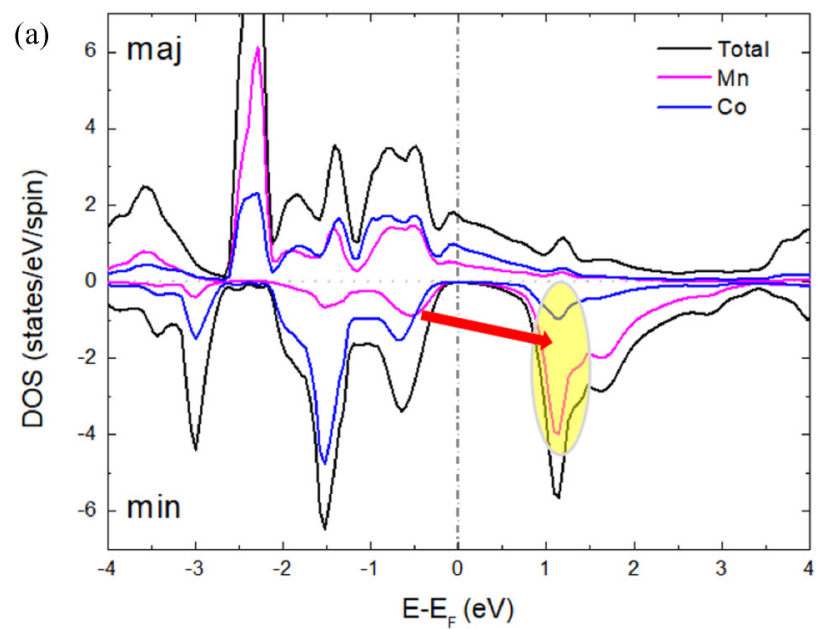

\section{Full Heusler: $\mathrm{Co}_{2} \mathrm{FeSi}$}

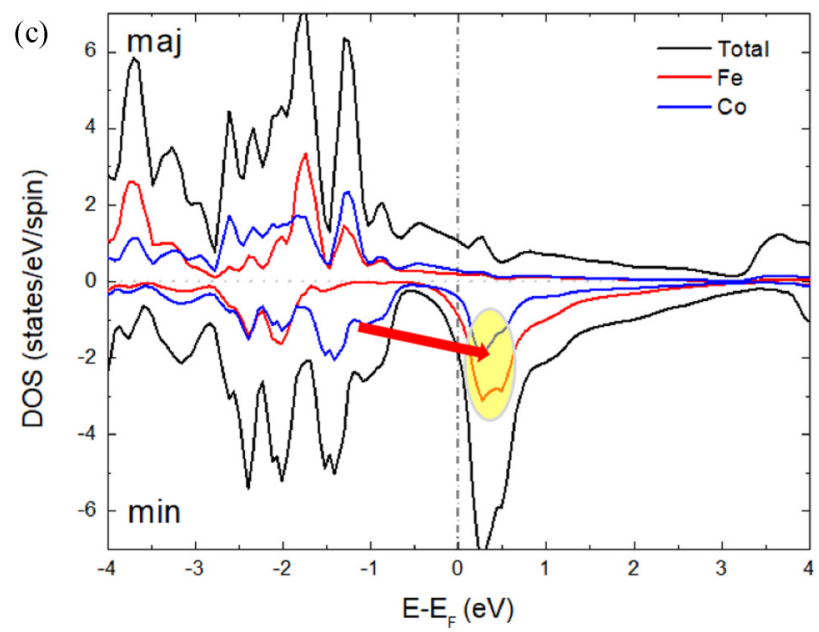

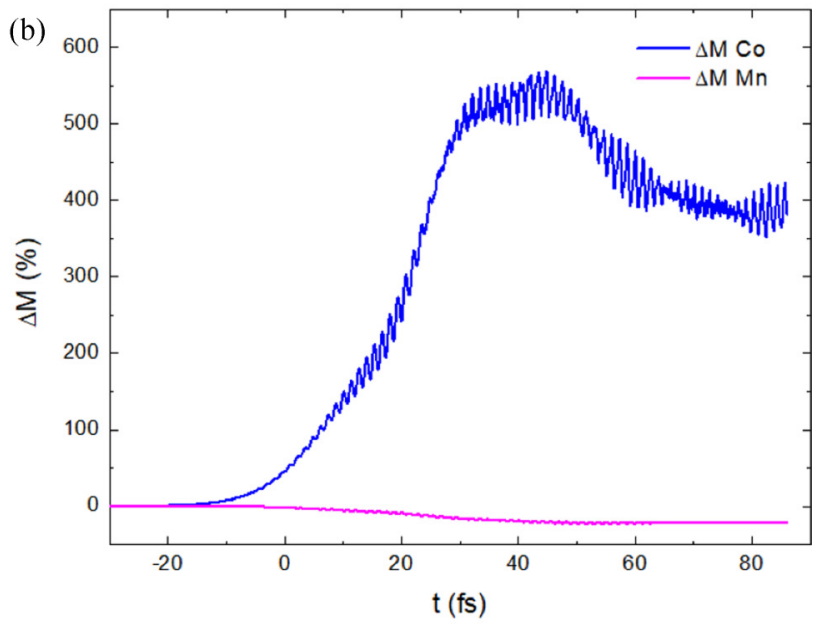

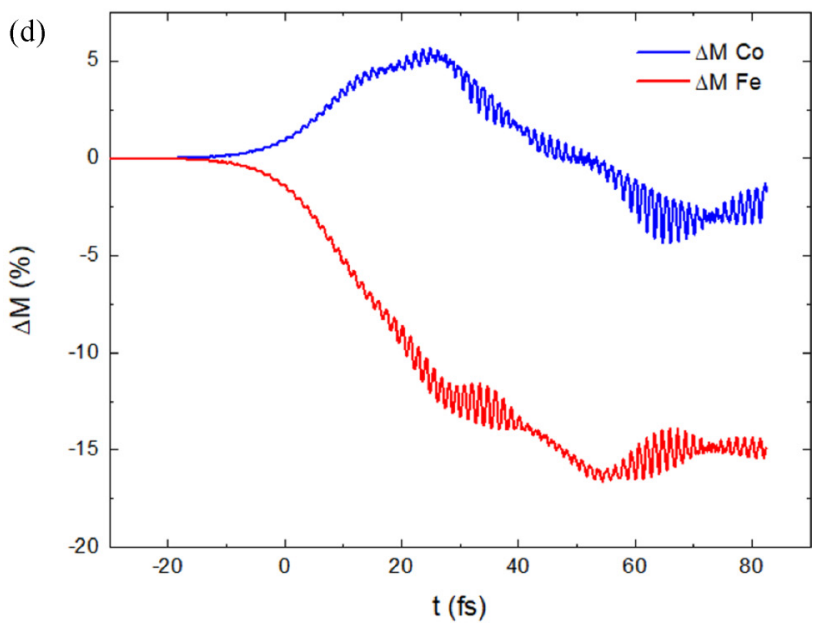

FIG. 2. (a), (c) Total and $d$-projected density of states (DOS) per atom for the half-Heusler $\mathrm{CoMnSb}_{\mathrm{Snd}}$ the full-Heusler $\mathrm{Co}_{2} \mathrm{FeSi}$. (b), (d) Relative change in magnetization after optical excitation for the Co and $\mathrm{Mn}$ sublattices in $\mathrm{CoMnSb}_{\mathrm{Sn}} \mathrm{Co}_{2} \mathrm{FeSi}$ from TD-DFT ( $T=0 \mathrm{~K}$, without consideration of temporal broadening and secondary relaxation processes, as present in the experimental data). The OISTR effect is much more pronounced in the half Heusler $(\Delta M \sim 550 \%)$ in comparison to the full Heusler $(\Delta M \sim 5 \%)$. The efficiency of OISTR can be deduced from the difference of the available $d$-projected DOS [marked in yellow in (a) and (c)], which is larger for the half-Heusler compound. In particular, the full-Heusler compound shows an increased density of Co states in the minority channel above the Fermi level $E_{\mathrm{F}}$ in comparison to the half Heusler, which is the main reason for the suppression of OISTR in experiment and theory. The red arrow indicates the dominant $1.5 \mathrm{eV}$ optical transitions from high DOS in the occupied regime to a high DOS in the unoccupied regime.

We therefore conclude that because of a smaller imbalance of the DOS between $\mathrm{Co}$ and Fe, respectively $\mathrm{Mn}$, and the presence of Co states above $E_{\mathrm{F}}$ in the minority channel (see the Supplemental Material [16]), OISTR is less efficient in the full Heuslers in comparison to the half Heuslers. We note that the absolute values of transferred magnetic moment per atom between the half- and full-Heusler compounds are within the same order of magnitude $\left(0.05-0.25 \mu_{\mathrm{B}}\right.$ /atom; see Fig. 3). However, due to the small initial magnetic moment of $\mathrm{Co} / \mathrm{Ni}$ for the half-Heusler systems, the relative change of the $\mathrm{Co} / \mathrm{Ni}$-sublattice magnetization is much more pronounced [up to $550 \%$; see Fig. 2(b)].

Hence, from our experimental and theoretical data, we see that a control of the composition of the Heusler compounds supports different efficiencies of OISTR. The main requirements for efficient OISTR are pronounced differences in the projected DOS between the two subsystems in an energy range that is driven by the optical excitation. In addition, it is necessary that the probability for different optical transitions, e.g., in the majority and minority channels, do not counteract each other with respect to spin transfer from one subsystem to another. Focusing on the former point, i.e., the DOS of the subsystems, a comprehensive theoretical study of the electronic structure of Co-based half and full Heuslers by Galanakis et al. [33] reveals that the decreased and increased minority density of Co states above the $E_{\mathrm{F}}$ is intrinsic to the formation of the gap in half and full Heuslers, respectively. In this manner, half Heuslers can generally be expected to show more efficient OISTR in comparison to full Heuslers.

Finally, we want to get back to the interpretation of our experimental data and in particular emphasize that it is not obvious why the time-resolved MOKE indeed shows 


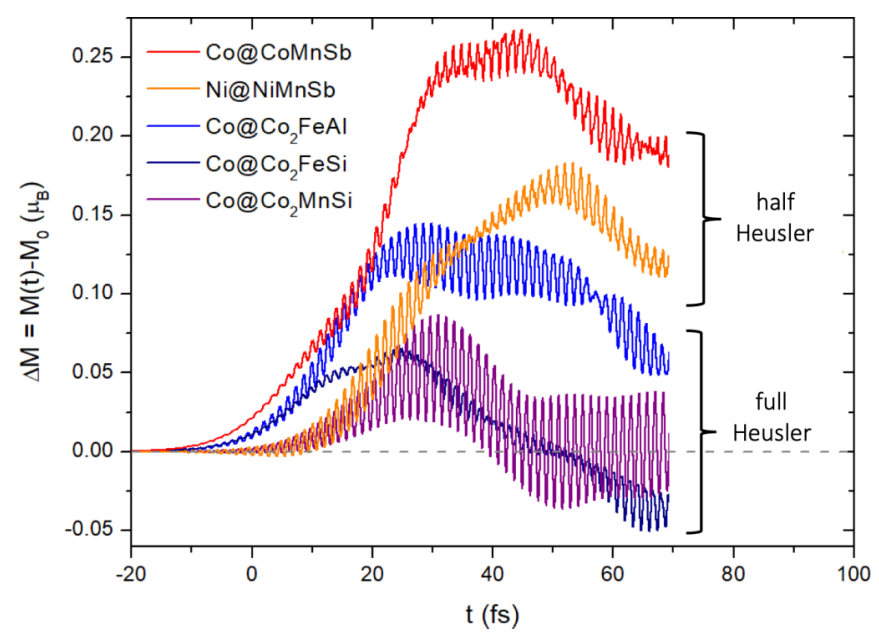

FIG. 3. Comparison of absolute OISTR moment in the half- and full-Heusler compounds from TD-DFT. Generally, OISTR is more efficient for the half Heuslers in comparison to the full Heuslers.

a pronounced OISTR signature, as we have seen for the half Heuslers [Fig. 1(a)]. MOKE in the visible wavelength range is generally regarded not to be element specific, as it is not trivially possible to disentangle different elemental contributions to the measured signal. Since OISTR does not change the total imbalance between majority and minority electrons in the material, one would expect MOKE to be rather insensitive to this process. If, however, the Kerr signal from one of the subsystems is dominant in the weighted average of the MOKE signal, which is possible and depends on the transitions probed in the Kerr process, element specificity becomes feasible (see, e.g., [34]). In our work, this is clearly the case for the half Heuslers, where the experimental data even resemble remarkably well the element-specific dynamics of the Co subsystem [Fig. 2(b)] of our theoretical calculations (see also theoretical confirmation of the sensitivity of MOKE to Co in the Supplemental Material [16]). Note, however, that even if MOKE is also more sensitive to a specific element in case of the full Heuslers, which might be the case, an increase would still be very hard to be observed experimentally. As discussed above, the relative magnetization change for the full Heuslers, which is the signal that is detected by time-resolved MOKE, is rather small in comparison to the half-Heusler compounds. In addition, secondary scattering processes induce a strong demagnetization, so that the small relative OISTR effect in the full Heusler can eventually not be detected with our visible time-resolved MOKE technique.

\section{CONCLUSION}

In summary, we have investigated the efficiency of the OISTR process for different compositions of Heusler compounds. We find experimentally and theoretically that the half Heuslers show a very strong signature of OISTR in contrast to the full Heuslers, where OISTR is much less efficient or even suppressed. We argue that for the systems studied here, the half Heuslers are ideal OISTR materials, because of a low DOS of $\mathrm{Co} / \mathrm{Ni}$ in the minority channel above $E_{\mathrm{F}}$, which is intrinsic to half Heuslers and different from full-Heusler compounds [33]. TD-DFT perfectly predicts the efficiency of OISTR, and can therefore ideally be used to guide experimental investigations that aim for coherent control of spin dynamics down to attosecond timescales.

\section{ACKNOWLEDGMENTS}

D.S. and S.M. acknowledge support from the DFG for funding through SFB1073 (Project No. A06). S.S. would like to thank the DFG for funding through TRR 227 (Project No. A04). P.E. would like to thank the DFG for funding through SPP 1840 (QUTIF). M.A., M.H., and M.C. acknowledge support from the SFB/TRR 173 "Spin + X" (Project No. A08).
[1] P. Elliott, T. Müller, J. K. Dewhurst, S. Sharma, and E. K. U. Gross, Ultrafast laser induced local magnetization dynamics in Heusler compounds, Sci. Rep. 6, 38911 (2016).

[2] J. K. Dewhurst, P. Elliott, S. Shallcross, E. K. U. Gross, and S. Sharma, Laser-induced intersite spin transfer, Nano Lett. 18, 1842 (2018).

[3] J. K. Dewhurst, S. Shallcross, E. K. U. Gross, and S. Sharma, Substrate-Controlled Ultrafast Spin Injection and Demagnetization, Phys. Rev. Appl. 10, 044065 (2018).

[4] M. Hofherr, S. Häuser, J. K. Dewhurst, P. Tengdin, S. Sakshath, H. T. Nembach, S. T. Weber, J. M. Shaw, T. J. Silva, H. C. Kapteyn, M. Cinchetti, B. Rethfeld, M. M. Murnane, D. Steil, B. Stadtmüller, S. Sharma, M. Aeschlimann, and S. Mathias, Ultrafast optically induced spin transfer in ferromagnetic alloys, Sci. Adv. 6, eaay8717 (2020).

[5] P. Tengdin, C. Gentry, A. Blonsky, D. Zusin, M. Gerrity, L. Hellbrück, M. Hofherr, J. Shaw, Y. Kvashnin, E. K. DelczegCzirjak, M. Arora, H. Nembach, T. J. Silva, S. Mathias, M. Aeschlimann, H. C. Kapteyn, D. Thonig, K. Koumpouras, O.
Eriksson, and M. M. Murnane, Direct light-induced spin transfer between different elements in a spintronic Heusler material via femtosecond laser excitation, Sci. Adv. 6, eaaz1100 (2020).

[6] F. Siegrist, J. A. Gessner, M. Ossiander, C. Denker, Y.-P. Chang, M. C. Schröder, A. Guggenmos, Y. Cui, J. Walowski, U. Martens, J. K. Dewhurst, U. Kleineberg, M. Münzenberg, S. Sharma, and M. Schultze, Light-wave dynamic control of magnetism, Nature (London) 571, 240 (2019).

[7] T. Graf, C. Felser, and S. S. Parkin, Simple rules for the understanding of Heusler compounds, Prog. Solid State Chem. 39, 1 (2011).

[8] C. Felser, L. Wollmann, S. Chadov, G. H. Fecher, and S. S. P. Parkin, Basics and prospective of magnetic Heusler compounds, APL Mater. 3, 041518 (2015).

[9] L. Wollmann, A. K. Nayak, S. S. Parkin, and C. Felser, Heusler 4.0: Tunable materials, Annu. Rev. Mater. Res. 47, 247 (2017).

[10] M. Djordjevic, G. Eilers, A. Parge, M. Münzenberg, and J. S. Moodera, Intrinsic and nonlocal Gilbert damping parameter in 
all optical pump-probe experiments, J. Appl. Phys. 99, 08F308 (2006).

[11] G. M. Müller, J. Walowski, M. Djordjevic, G.-X. Miao, A. Gupta, A. V. Ramos, K. Gehrke, V. Moshnyaga, K. Samwer, J. Schmalhorst, A. Thomas, A. Hütten, G. Reiss, J. S. Moodera, and M. Münzenberg, Spin polarization in half-metals probed by femtosecond spin excitation, Nat. Mater. 8, 56 (2008).

[12] J. Walowski, M. D. Kaufmann, B. Lenk, C. Hamann, J. McCord, and M. Münzenberg, Intrinsic and non-local Gilbert damping in polycrystalline nickel studied by Ti:sapphire laser fs spectroscopy, J. Phys. D: Appl. Phys. 41, 164016 (2008).

[13] J.-P. Wuestenberg, D. Steil, S. Alebrand, T. Roth, M. Aeschlimann, and M. Cinchetti, Ultrafast magnetization dynamics in the half-metallic Heusler alloy $\mathrm{Co}_{2} \mathrm{Cr}_{0.6} \mathrm{Fe}_{0.4} \mathrm{Al}$, Phys. Status Solidi B 248, 2330 (2011).

[14] D. Steil, O. Schmitt, R. Fetzer, T. Kubota, H. Naganuma, M. Oogane, Y. Ando, A. K. Suszka, O. Idigoras, G. Wolf, B. Hillebrands, A. Berger, M. Aeschlimann, and M. Cinchetti, Ultrafast magnetization dynamics in Co-based Heusler compounds with tuned chemical ordering, New J. Phys. 16, 063068 (2014).

[15] D. Steil, O. Schmitt, R. Fetzer, T. Kubota, H. Naganuma, M. Oogane, Y. Ando, S. Rodan, C. G. F. Blum, B. Balke, S. Wurmehl, M. Aeschlimann, and M. Cinchetti, Impact of local order and stoichiometry on the ultrafast magnetization dynamics of Heusler compounds, J. Phys. D: Appl. Phys. 48, 164016 (2015).

[16] See Supplemental Material at http://link.aps.org/supplemental/ 10.1103/PhysRevResearch.2.023199 for the full set of DFT and TD-DFT data on the Heusler compounds, calculations of the MOKE signal change due to OISTR, as well as information on the sample systems.

[17] E. Runge and E. K. U. Gross, Density-Functional Theory for Time-Dependent Systems, Phys. Rev. Lett. 52, 997 (1984).

[18] P. Elliott, F. Furche, and K. Burke, Excited states from timedependent density functional theory, in Reviews in Computational Chemistry (John Wiley \& Sons, 2009), Chap. 3, pp. $91-165$.

[19] C. A. Ullrich, Time-Dependent Density-Functional Theory: Concepts and Applications, Oxford Graduate Texts (Oxford University Press, Oxford, 2011).

[20] S. Sharma, J. K. Dewhurst, and E. K. U. Gross, Optical response of extended systems using time-dependent density functional theory, in First Principles Approaches to Spectroscopic Properties of Complex Materials, edited by C. Di Valentin, S. Botti, and M. Cococcioni (Springer, Berlin, 2014), pp. 235-257.

[21] K. Krieger, J. K. Dewhurst, P. Elliott, S. Sharma, and E. K. U. Gross, Laser-induced demagnetization at ultrashort time scales: Predictions of TDDFT, J. Chem. Theory Comput. 11, 4870 (2015)

[22] J. K. Dewhurst, K. Krieger, S. Sharma, and E. K. Gross, An efficient algorithm for time propagation as applied to linearized augmented plane wave method, Comput. Phys. Commun. 209, 92 (2016).

[23] J. K. Dewhurst et al., The Elk Code, http://elk.sourceforge.net.

[24] J. Kubler, K.-H. Hock, J. Sticht, and A. R. Williams, Density functional theory of non-collinear magnetism, J. Phys. F: Met. Phys. 18, 469 (1988).

[25] B. Koopmans, G. Malinowski, F. Dalla Longa, D. Steiauf, M. Fähnle, T. Roth, M. Cinchetti, and M. Aeschlimann, Explaining the paradoxical diversity of ultrafast laser-induced demagnetization, Nat. Mater. 9, 259 (2010).

[26] M. Battiato, K. Carva, and P. M. Oppeneer, Superdiffusive Spin Transport as a Mechanism of Ultrafast Demagnetization, Phys. Rev. Lett. 105, 027203 (2010).

[27] E. Turgut, C. La-o-vorakiat, J. M. Shaw, P. Grychtol, H. T. Nembach, D. Rudolf, R. Adam, M. Aeschlimann, C. M. Schneider, T. J. Silva, M. M. Murnane, H. C. Kapteyn, and S. Mathias, Controlling the Competition between Optically Induced Ultrafast Spin-Flip Scattering and Spin Transport in Magnetic Multilayers, Phys. Rev. Lett. 110, 197201 (2013).

[28] S. Eich, M. Plötzing, M. Rollinger, S. Emmerich, R. Adam, C. Chen, H. C. Kapteyn, M. M. Murnane, L. Plucinski, D. Steil, B. Stadtmüller, M. Cinchetti, M. Aeschlimann, C. M. Schneider, and S. Mathias, Band structure evolution during the ultrafast ferromagnetic-paramagnetic phase transition in cobalt, Sci. Adv. 3, 1602094 (2017).

[29] D. Steil, S. Alebrand, T. Roth, M. Krauß, T. Kubota, M. Oogane, Y. Ando, H. C. Schneider, M. Aeschlimann, and M. Cinchetti, Band-Structure-Dependent Demagnetization in the Heusler Alloy $\mathrm{Co}_{2} \mathrm{Mn}_{1-x} \mathrm{Fe}_{x} \mathrm{Si}$, Phys. Rev. Lett. 105, 217202 (2010).

[30] A. Mann, J. Walowski, M. Münzenberg, S. Maat, M. J. Carey, J. R. Childress, C. Mewes, D. Ebke, V. Drewello, G. Reiss, and A. Thomas, Insights into Ultrafast Demagnetization in Pseudogap Half-Metals, Phys. Rev. X 2, 041008 (2012).

[31] Y. Liu, L. R. Shelford, V. V. Kruglyak, R. J. Hicken, Y. Sakuraba, M. Oogane, and Y. Ando, Optically induced magnetization dynamics and variation of damping parameter in epitaxial $\mathrm{Co}_{2} \mathrm{MnSi}$ Heusler alloy films, Phys. Rev. B 81, 094402 (2010).

[32] Z. Zhang, D. Wu, Z. Luan, H. Yuan, Z. Zhang, J. Zhao, H. Zhao, and L. Chen, Photoinduced spin precession and ultrafast demagnetization in $\mathrm{Co}_{2} \mathrm{FeAl}$ films with crossover from in-plane to perpendicular magnetic easy axis, IEEE Magn. Lett. 6, 3500604 (2015).

[33] I. Galanakis, P. Mavropoulos, and P. H. Dederichs, Electronic structure and Slater-Pauling behavior in half-metallic Heusler alloys calculated from first principles, J. Phys. D: Appl. Phys. 39, 765 (2006).

[34] A. R. Khorsand, M. Savoini, A. Kirilyuk, A. V. Kimel, A. Tsukamoto, A. Itoh, and T. Rasing, Element-Specific Probing of Ultrafast Spin Dynamics in Multisublattice Magnets with Visible Light, Phys. Rev. Lett. 110, 107205 (2013). 